\title{
Nasal Stick Dosage Form
}

National Cancer Institute

\section{Source}

National Cancer Institute. Nasal Stick Dosage Form. NCI Thesaurus. Code C149690.

Solid single-dose preparation, usually rod-shaped or conical, intended for nasal use to obtain a local effect. 